\title{
Lance-Adams Syndrome Treated by Perampanel in the Acute Term
}

\author{
Masahito Katsuki $^{1}$, Norio Narita ${ }^{2}$, Iori Yasuda ${ }^{2}$, Teiji Tominaga ${ }^{1}$ \\ 1. Neurosurgery, Tohoku University, Sendai, JPN 2. Neurosurgery, Kesennuma City Hospital, Kesennuma, JPN
}

Corresponding author: Norio Narita, naritanorio1111@gmail.com

\begin{abstract}
Lance-Adams syndrome (LAS) is chronic post-hypoxic myoclonus after a hypoxic encephalopathy. Recently, the report on LAS in the chronic term treated by perampanel (PER) is increasing. However, PER's efficacy in the "acute term" has not been reported. Here, we report an LAS patient who markedly improved when PER was added to his existing treatment regime in the acute term. The 65 -year-old patient presented with a return of spontaneous circulation after cardiopulmonary arrest. He developed myoclonus on the admission day, and it led to tonic-clonic convulsion. We started levetiracetam $3000 \mathrm{mg} /$ day, lacosamide $400 \mathrm{mg} /$ day, general anesthesia using midazolam $180 \mathrm{mg} /$ day, dexmedetomidine $1000 \mu \mathrm{g} / \mathrm{day}$, and fentanyl $1.2 \mathrm{mg} /$ day. We could stop the convulsions after $18 \mathrm{~h}$ from the onset. We tried to reduce sedatives, but his convulsion recurred. We added PER $2 \mathrm{mg}$ /day for three days, PER $4 \mathrm{mg} /$ day for next four days, then used PER $8 \mathrm{mg} /$ day and we could gradually reduce the sedatives. Single-photon emission computed tomography on day 40 showed cerebral blood flow (CBF) increase at the bilateral anterior lobes of the cerebellum, medial temporal lobes, and supplementary motor and premotor areas, while CBF decrease at the brain surface of the frontal, parietal, and temporal lobes. The myoclonus disappeared since day 12 , and he was transferred to another rehabilitation hospital on day 56 . The optimal treatment strategy has not been established for LAS, but our case suggested that PER could be one of the choices to treat LAS in the acute term.
\end{abstract}

Review began 03/03/2021 Review ended 03/06/2021 Published 03/08/2021

๑) Copyright 2021 Katsuki et al. This is an open access article distributed under the terms of the Creative Commons Attribution License CC-BY 4.0., which permits unrestricted use, distribution, and reproduction in any medium, provided the original author and source are credited.
Categories: Cardiology, Neurology, Neurosurgery

Keywords: cardiopulmonary arrest, hypoxia, lance-adams syndrome, myoclonus, n-isopropyl-p-[123i]iodoamphetamine single-photon emission computed tomography, perampanel, 3-dimensional stereotactic surface projections

\section{Introduction}

Lance-Adams syndrome (LAS) is chronic post-hypoxic myoclonus with onset days to weeks after a hypoxic encephalopathy [1,2]. LAS originated from a 1963 report in which four patients with acute hypoxic encephalopathy due to cardiac arrest after anesthesia or postoperative airway obstruction developed generalized myoclonus during recovery from coma [2]. The LAS's myoclonus is variably associated with dysarthria, ataxia, seizures, or cognitive deficits [1]. Given LAS's relative rarities, there are no controlled treatment studies of LAS. The majority of cases require polypharmacy management or general anesthesia therapy, with an incomplete response [1].

Recently, the report on LAS in the chronic term treated by perampanel (PER) is increasing [3-8]. However, PER's efficacy in the acute term has not been reported. Here, we report an LAS patient who markedly improved when PER was added to his existing treatment regime in the acute term.

\section{Case Presentation}

A 65-year-old man, who did not have any past histories, suddenly fainted. He went into cardiopulmonary arrest (CPA) with bystanders, but the bystanders did not perform resuscitation. After 11 minutes, the ambulance and rescue team arrived and started resuscitation. After five cycles of chest compressions and rescue breaths at a ratio of 30:2, a return of spontaneous circulation was achieved, and the patient arrived at our hospital. His blood pressure was $158 / 108 \mathrm{mmHg}$, his heart rate $122 \mathrm{bpm}$, respiratory rate 15/min, blood temperature $36.8^{\circ} \mathrm{C}$, and saturation of percutaneous oxygen $92 \%$ with $10 \mathrm{~L} / \mathrm{min}$ oxygen. His consciousness was as Japan Coma Scale (JCS) 200 and Glasgow Coma Scale (GCS) 3 (E1VTM1). The pupil sizes were 5 mm equally in both eyes, and the eyes were roving. Laboratory tests reveal slight changes after resuscitation, but nothing related to sudden CPA. Echocardiography, electrocardiography, and coronary angiography did not suggest cardiologic diseases. Head computed tomography (CT) angiography and magnetic resonance imaging (MRI) also did not show any findings related to the CPA. The cause of CPA was unknown, and general care with ventilator and vasopressors was started.

After 4 hours from the onset, he presented $1 \mathrm{~Hz}$ left upper extremities' myoclonus seizure intermittently lasting for around 3 minutes, and we started levetiracetam (LEV) $3000 \mathrm{mg} / \mathrm{day}$, but the myoclonus could not be stopped. After 16 hours from the onset, we added lacosamide (LCM) $400 \mathrm{mg}$ /day. However, his myoclonus continued. The next day, he developed tonic-clonic convulsions. We started general anesthesia with midazolam $180 \mathrm{mg} /$ day, dexmedetomidine $1000 \mu \mathrm{g} /$ day, and fentanyl $1.2 \mathrm{mg} /$ day with adequate 


\section{Cureus}

noradrenaline and dobutamine for about $48 \mathrm{~h}$. Finally, we could stop the convulsions. We tried to reduce sedatives, but his convulsion or myoclonus recurred. Therefore, we added PER $2 \mathrm{mg} / \mathrm{day}$ on day 3 . We performed a tracheostomy on day 5 . On day 7 , we prescribed PER $4 \mathrm{mg} /$ day because we could not reduce the sedatives. We then used PER $8 \mathrm{mg} /$ day from day 12, and we could gradually reduce the sedatives. Because of the circulatory failure and sepsis, we carefully and slowly reduced sedatives and vasopressors' dosage. On day 38, we finally stopped sedatives and vasopressors. On day 39, we performed CT and MRI including diffusion tensor imaging, but they did not reveal any characteristic findings. On day $40, \mathrm{~N}$-isopropyl-p[123I]-iodoamphetamine single-photon emission computed tomography (123I-IMP-SPECT) was performed and evaluated by 3-dimensional stereotactic surface projections (3D-SSP) standardized by the whole brain [9]. The cerebral blood flow (CBF) increased at the bilateral anterior lobes of the cerebellum, medial temporal lobes, and supplementary motor and premotor areas; while CBF decreased at the brain surface of the frontal, parietal, and temporal lobes (Figure 1). We performed electroencephalography (EEG), but only noise due to alternating current was detected despite using an electromagnetic noise prevention sheet and thiopental sedation. His consciousness improved as JCS 3 and GCS 10 (E4VTM6), and rehabilitation was continued using a wheelchair. The myoclonus has disappeared since day 12 , and he was transferred to another rehabilitation hospital on day 56 because he is from another prefecture.

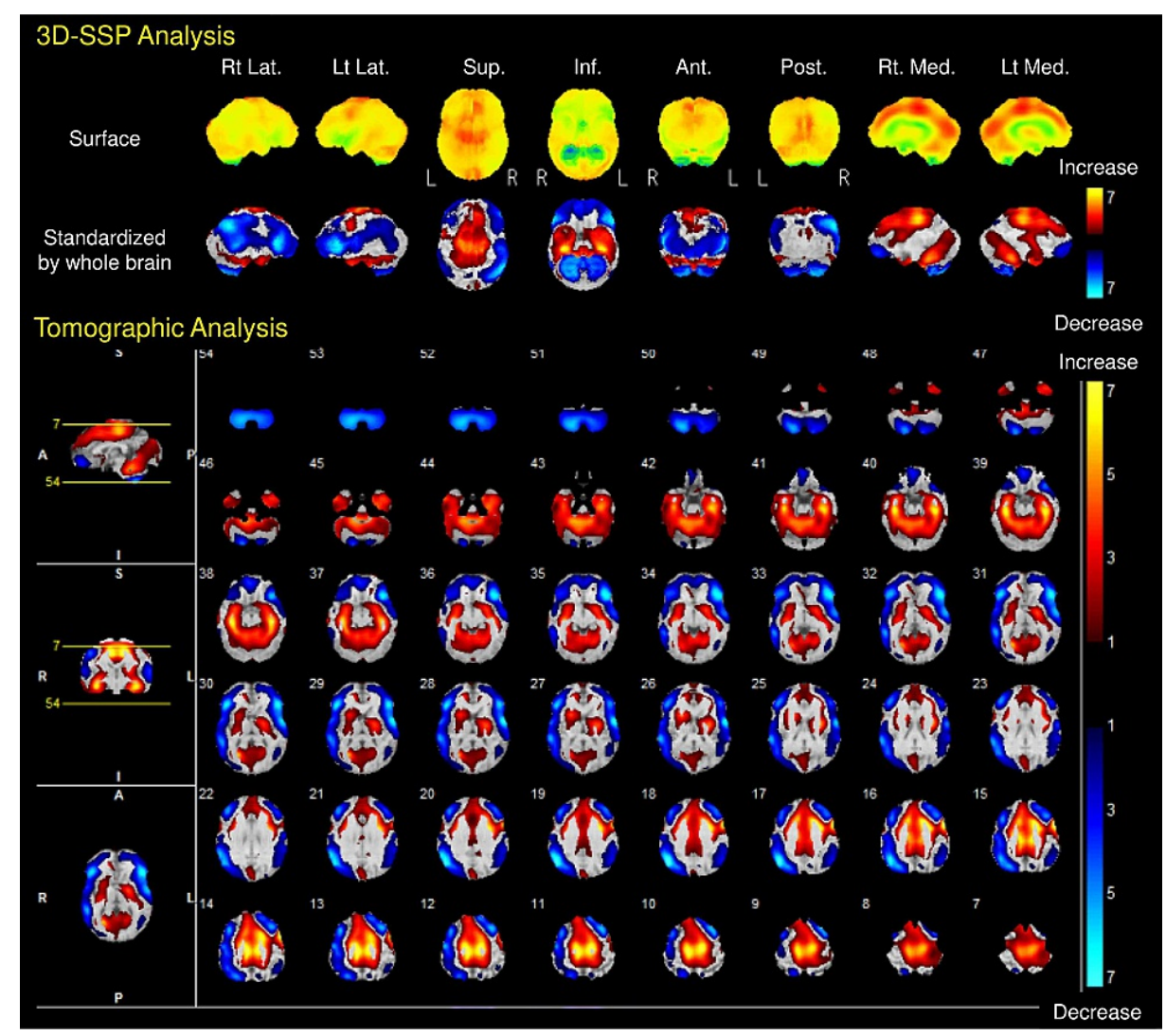

FIGURE 1: N-isopropyl-p-[123I]-iodoamphetamine single-photon emission computed tomography evaluated by 3-dimensional stereotactic surface projections (3D-SSP) standardized by the whole brain

The cerebral blood flow (CBF) increased at the bilateral anterior lobes of the cerebellum, medial temporal lobes, and supplementary motor and premotor areas; while CBF decreased at the brain surface of the frontal, parietal, and temporal lobes.

Ant: anterior; Inf: inferior; Lat: lateral; Med: medial; Post: posterior; Sup: superior.

\section{Discussion}

There are no well-defined guidelines for treatment in LAS. Our case presented status epilepticus started from myoclonic seizures on the admission day. We used multiple antiepileptic drugs and performed general anesthesia therapy according to the Japanese guideline [10]. We could not reduce sedative drugs without PER, but after adding PER, the patient was extubated and started rehabilitation. Although the general anesthesia therapy's effect should be considered, our case suggested the efficacy of PER for LAS's myoclonic seizures and subsequent status epilepticus as the acute treatment choice. 


\section{Lance-Adams syndrome}

The LAS's myoclonic seizure mechanism is unclear but hypothesized to be originated from the cortex, and the myoclonus may relate to abnormal gamma-aminobutyric acid (GABA) and serotonin neurotransmission in the brain $[1,4,8]$. We did not evaluate GABA or serotonin levels in serum and cerebrospinal fluid, because we could not order these tests in our hospital. However, we speculate that the patient's GABA and serotonin neurotransmission system is dysregulated as previously reported hypothesis.

Previous reports describe that SPECT of LAS patients showed decreased blood flow in the right basal ganglia and left temporal lobe $[11,12]$. Ours showed CBF increase at the bilateral supplementary motor and premotor areas as well as CBF decrease at the brain surface of the frontal, parietal, and temporal lobes (Figure 1). This is different findings compared to the previous reports because the region where the $\mathrm{CBF}$ was elevated was different from the previous reports $[11,12]$. Previous functional imaging study demonstrated increased glucose metabolism in the ventrolateral thalamus, pontine tegmentum, and medial temporal lobes, suggesting an involvement of the basal ganglia-thalamo-cortical network [13,14]. The ventrolateral thalamus connects to the motor cortex, premotor cortex, and supplementary motor cortex [15]. These cortexes play important roles in initiating motor function. Our SPECT results suggested that CBF might increase at the medial frontal lobes due to increased glucose metabolism in the ventrolateral thalamus, causing myoclonus. CBF decrease at the whole brain surface may be due to results of hypoxia. Further CBF and functional studies are needed.

There are no typical findings of LAS's EEG $[1,11]$. In the literature review, burst suppression was the most common pattern, while alpha coma was the least common pattern [1]. We performed EEG, but only noise due to alternating current was detected despite using an electromagnetic noise prevention sheet and thiopental sedation. This might be a new characteristic EEG finding of LAS, but further studies are needed.

LAS's somatosensory evoked potentials (SEP) sometimes show characteristic findings. The early SEP component is high amplitude so-called a giant SEP. Then, C-reflex, which records muscle activity at long latency after electrical stimulation, can be detected $[4,8]$. Oi et al. showed a positive correlation between PER blood concentration and prolongation of latency, such as P25 in giants SEP, which can be used as a potential biomarker for assessing the objective effects of PER on intractable cortical myoclonus [8]. We did not perform SEP evaluation because we do not have these instruments nor neurologist who could evaluate the results, and we should have performed it before and after PER prescription to evaluate PER's efficacy.

\section{Perampanel}

Perampanel (PER) is a selective noncompetitive $\alpha$-amino-3-hydroxy-5-methyl-4-isoxazole propionic acid (AMPA) receptor antagonist and inhibits excessive excitation of nerve cells by reducing Ca2+ inflow. PER was recently introduced as adjunctive therapy for patients with epilepsy $[4,8,16]$. The pharmacological characteristics of PER - (1) dose-dependent reduction of seizure and myoclonus [17]; (2) possible threshold dosage, e.g., $6 \mathrm{mg} /$ day (maximum dose of PER is generally $12 \mathrm{mg} /$ day) for severe side effects, such as irritability, anxiety, violence, hallucination, weight gain [18]; (3) very slow titration was recommended to avoid side effects [16] - are reported. While PER shows promise as an antiepileptic drug with a new mechanism, it must be used cautiously due to side effects. In our case, the myoclonus and the subsequent status epilepticus could be stopped by using $8 \mathrm{mg} /$ day PER with a gradual increase in the short term, but we should pay attention to side effects. We will reduce PER to around 2-6 mg/day, according to the previous report $[8,18]$.

Table 1 shows the previously reported LAS treated by PER. In all previous reports, PER is prescribed for chronic LAS, and their myoclonus or activities in daily livings were improved [3-8]. We prescribed PER for LAS in the acute phase, and we could stop sedation and reach extubation. It is unknown whether the status epilepticus resolved by prolonged sedation or PER, but our case suggested that PER could be one of acute treatments for LAS. Further studies are desired on LAS and PER. 


\section{Cureus}

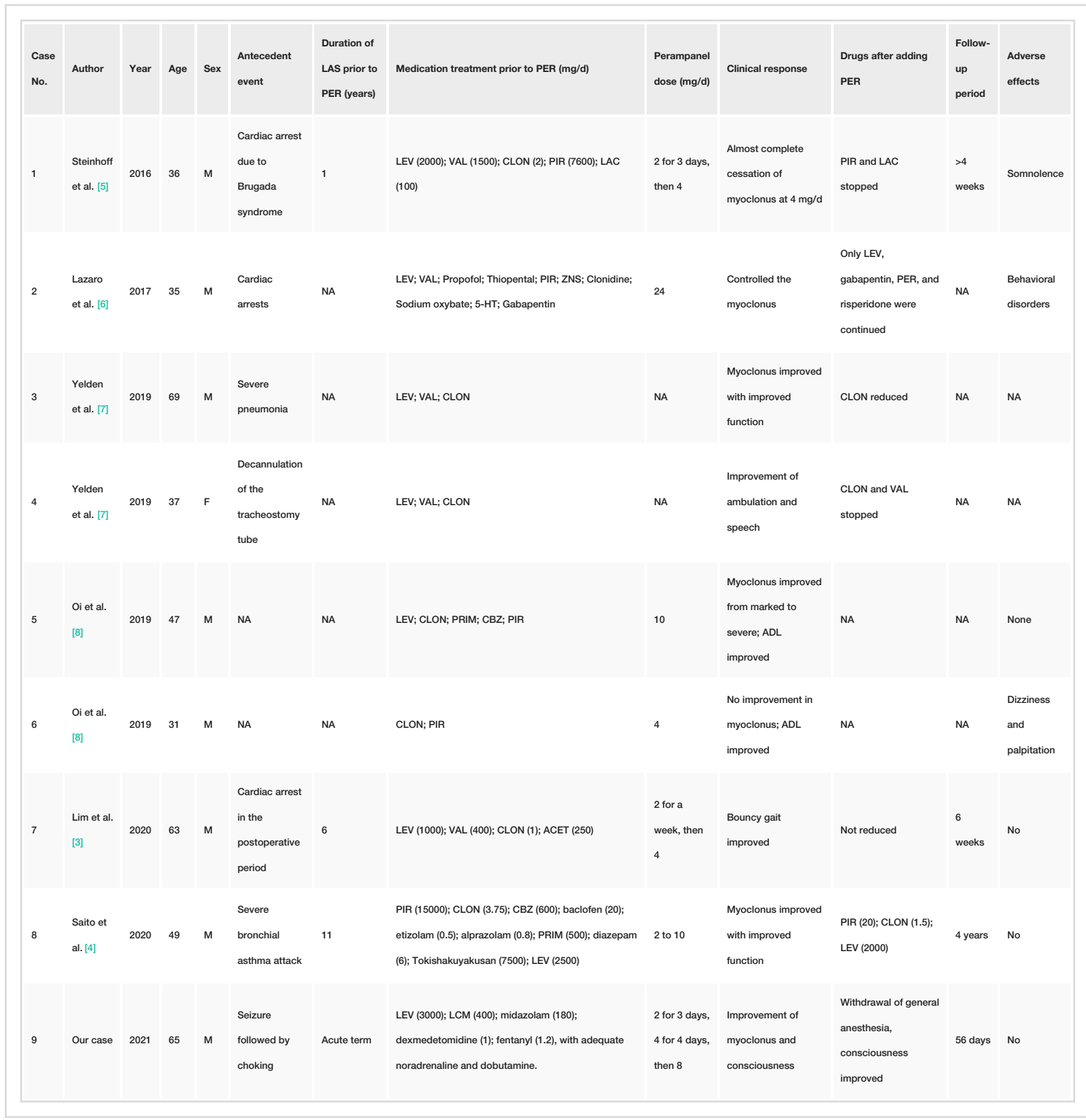

TABLE 1: Previous reports of Lance-Adams syndrome patients treated with perampanel

ACET: Acetazolamide; CBZ: Carbamazepine; CLON: Clonazepam; LAC: Lacosamide; LEV: Levetiracetam; NA: Not Available; PER: Perampanel; PIR: Piracetam; PRIM: Primidone; VAL: Sodium valproate; ZNS: Zonisamide; 5-HT: 5-hydroxytryptophan.

\section{Conclusions}

We herein reported an LAS patient who improved his myoclonus and subsequent status epilepticus markedly when PER was added to his existing treatment regime in the acute term. The optimal treatment strategy has not been established, but our case suggested that PER could be one of the choices to treat LAS in the acute term.

\section{Additional Information \\ Disclosures}

Human subjects: Consent was obtained or waived by all participants in this study. Conflicts of interest: In compliance with the ICMJE uniform disclosure form, all authors declare the following: Payment/services info: All authors have declared that no financial support was received from any organization for the submitted work. Financial relationships: All authors have declared that they have no financial relationships at present or within the previous three years with any organizations that might have an 
interest in the submitted work. Other relationships: All authors have declared that there are no other relationships or activities that could appear to have influenced the submitted work.

\section{Acknowledgements}

Thank you for the cooperation for this article by Siqi Cai for data acquisition. He is the resident of our hospital.

\section{References}

1. Gupta HV, Caviness JN: Post-hypoxic myoclonus: current concepts, neurophysiology, and treatment . Tremor Other Hyperkinet Mov (NY). 2016, 6:409. 10.7916/D89C6XM4

2. Lance JW, Adams RD: The syndrome of intention or action myoclonus as a sequel to hypoxic encephalopathy. Brain. 1963, 86:111-136. 10.1093/brain/86.1.111

3. Lim S-Y, Jasti DB, Tan AH: Improvement of "bouncy gait" in Lance-Adams syndrome with perampanel . Cureus. 2020, 12 :e6773. 10.7759/cureus.6773

4. Saito K, Oi K, Inaba A, Kobayashi M, Ikeda A, Wada Y: A case of the successful treatment of severe myoclonus with Lance-Adams syndrome by add-on perampanel showing long term effects. (Article in Japanese). Rinsho Shinkeigaku. 2021, 61:18-23. 10.5692/clinicalneurol.cn-001477

5. Steinhoff BJ, Bacher M, Kurth C, Staack AM, Kornmeier R: Add-on perampanel in Lance-Adams syndrome. Epilepsy Behav Case Rep. 2016, 6:28-29. 10.1016/j.ebcr.2016.05.001

6. Lazaro López E, Jalon Urbina MM, Sánchez AP, et al.: DI-096 refractory Lance-Adams syndrome: pharmacotherapy management and iatrogenic complications. Eur J Hosp Pharm. 2017, 24:A156. 10.1136/ejhpharm-2017-000640.343

7. Yelden K, Rendell L, Vardy L, Rose A, Crilly S, Merrison K: Treatment of refractory myoclonus with perampanel in Lance Adams syndrome: a report of two cases. Accepted abstracts from the International Brain Injury Association's 13th World Congress on Brain Injury. Brain Inj. 2019, 33:1-337. 10.1080/02699052.2019.1608749

8. Oi K, Neshige S, Hitomi T, et al.: Low-dose perampanel improves refractory cortical myoclonus by the dispersed and suppressed paroxysmal depolarization shifts in the sensorimotor cortex. Clin Neurophysiol. 2019, 130:1804-1812. 10.1016/j.clinph.2019.07.006

9. Minoshima S, Frey KA, Koeppe RA, Foster NL, Kuhl D: A diagnostic approach in Alzheimer’s disease using three-dimensional stereotactic surface projections of fluorine-18-FDG PET. J Nucl Med. 1995, 36:1238-1248.

10. Japanese Society of Neurology: Clinical Practice Guideline for Epilepsy 2018. Igaku Shoin, Tokyo; 2018.

11. Lee HL, Lee JK: Lance-Adams syndrome. Ann Rehabil Med. 2011, 35:939-943. 10.5535/arm.2011.35.6.939

12. Zhang YX, Liu JR, Jiang B, et al.: Lance-Adams syndrome: a report of two cases. J Zhejiang Univ Sci B. 2007, 8:715-720. 10.1631/jzus.2007.B0715

13. Frucht S, Trošt M, Ma Y, Eidelberg D: The metabolic topography of posthypoxic myoclonus . Neurology. 2004, 62:1879-1881. 10.1212/01.WNL.0000125336.05001.23

14. Ramdhani RA, Frucht SJ, Kopell BH: Improvement of post-hypoxic myoclonus with bilateral pallidal deep brain stimulation: a case report and review of the literature. Tremor Other Hyperkinet Mov (NY). 2017, 7:461. 10.7916/D8NZ8DXP

15. Katsuki M, Narisawa A, Karibe H, Kameyama M, Tominaga T: Mutism resulting from heterochronic bilateral cerebellar hemorrhages - A case report. Surg Neurol Int. 2019, 10:122. 10.25259/SNI-18-2019

16. Hanada T, Hashizume Y, Tokuhara N, et al.: Perampanel: a novel, orally active, noncompetitive AMPAreceptor antagonist that reduces seizure activity in rodent models of epilepsy. Epilepsia. 2011, 52:13311340. 10.1111/j.1528-1167.2011.03109.x

17. Trinka E, Steinhoff BJ, Nikanorova M, Brodie MJ: Perampanel for focal epilepsy: insights from early clinical experience. Acta Neurol Scand. 2016, 133:160-172. 10.1111/ane.12529

18. Crespel A, Gelisse P, Tang NPL, Genton P: Perampanel in 12 patients with Unverricht-Lundborg disease . Epilepsia. 2017, 58:543-547. 10.1111/epi.13662 